\title{
Diagenesis of Xing'anling Oil Reservoir in Sudeerte Area
}

\author{
Yu Haoran ${ }^{1}$, Yu Xuntao ${ }^{1 *}$, liu Chengzhi ${ }^{1}$, Yuan Hongqi ${ }^{1}$ \\ ${ }^{1}$ Northeast Petroleum University, School of Earth Sciences, 163318
}

\begin{abstract}
In order to explore the types of diagenesis in Sudeerte area, through core and thin slice identification, it is clear that the Xing'anling oil layer is mainly affected by compaction, cementation, andrecrystallization. The compaction is mainly early compaction, late pressure dissolution is not obvious; cementation presents two forms of crystalline quartz and amorphous opal; recrystallization is manifested as the conversion process of opal t-ochalcedony quartz.
\end{abstract}

\section{Regional geology and stratigraphic characteristics}

The Hailar Basin base is composed of Indosinian granites and Budate Group in the west of the Herzegovina. The basin is filled with Jurassic,Cretaceous, Tertiary and Quaternary bottom layers. The bottom of the Jurassic and Cretaceous are the main deposits in the basin, from bottom to top:the upper Jurassic Xing'anling oil formation volcanic rock intercalation, the lower Cretaceous Tongbomiao Formation, Nantun Formation,Damoguaihe Formation,Yimin Formation and Qingyuangang Formation, Upper Cretaceous Beier Lake Group Qingyuangang Formation, Upper Tertiary Huchashan Formation and Quaternary ${ }^{[1]}$. The Sudeerte Oilfield is located in the Sudeerte structural belt of Beier Depression, Beier Lake depression,Hailar Basin. The faults arranged in geese in the west gradually transition to the Beixi syncline, and several faults to the east gradually enter the Beizhong syncline. The distribution direction of the faults in the zone is roughly northeast and northeast east,with large cross-section width, large and rapid change of fault distance,large faults can generally overlap, small faults in the area are also very developed,and most of the traps in the area are blocked by faults, In the fault blocks formed,most of the eastern faults spread in a geese pattern $^{[2]}$. The main oil layers developed in the Sudeerte oil field are the Xing'anling oil layer and the Budate group oil layer. The main oil layers are located in the Sudeert fault uplift zone, which is mainly divided into multiple large fault blocks by the northeast and near east-west faults. Northeast.The central fault block is further cut by the NE and NE trending internal faults, forming an extremely complex fault system ${ }^{[3]}$.According to the section and plane characteristics of the fault,from west to east,the fault appears as obvious "graben","horst" and "fault step","Y" and other forms. The laps are spread at an acute angle,and the eastern faults are mostly spread in geese,reflecting the result of the left-handed stress field ${ }^{[4-}$
5]. The reservoirs are mainly composed of conglomeratebearing sandstone and lithic sandstone, mostly with tophaceous. The structural maturity and composition maturity of sandstone are low. There are many types of pores, including intergranular pores, intragranular pores, micro-cracks and secondary dissolution pores, and the reservoir space is dominated by intergranular pores. The micro-cracks and secondary dissolution pores are also relatively developed, the micro-cracks are not filled, and the connectivity is good. Most of the feldspar and cuttings particles are partially dissolved, forming secondary dissolved pores.

\section{Petrological characteristics}

At different diagenetic stages, the composition, grain composition, grain structure of the rock will be different ${ }^{[6-}$ ${ }^{7]}$.Microscopic identification and analysis show that the particles in the rocks in this area are mostly explosive quartz crystal debris, tuff debris and glass debris, with a content of $40-50 \%$ or even higher; the roundness is poor and belongs to pyroclastic sedimentary rock class. The lithology of most samples is named higher; the sandstone and tophaceous mudstone; those with less tophaceous components are classified as tuff-containing mudstone. The interstitial materials are mainly glass debris and small rock debris; mostly siliceous cement formed by glass debris devitrification. Some samples are completely siliceous cemented, some are semi-cemented, and the unbonded part becomes pores.

Sandstone types are mainly feldspar lithic sandstone, quartz feldspar lithic sandstone, etc. Most of the quartz particles in sandstone have a particle size between 0.1 and $0.5 \mathrm{~mm}$, and the content of quartz is $10-15 \%$. The quartz is sub-angular-sub-circular, poorly sorted-medium, with low structural maturity. The size of debris and feldspar varies, Mainly coarse sandstone, medium sandstone, and fine sandstone, with less siltstone or argillaceous siltstone. The roundness of detrital particles is poor. The cuttings and feldspar are mostly sub-angular. The content of

*Corresponding author's e-mail: yxt0407@163.com 
feldspar is $10 \%-15 \%$. In the composition of sandstone, the content of unstable components such as cuttings is high, reaching about $70 \%$, The ingredient maturity is low. The miscellaneous base fills part of the pores, and carbonate cements can be seen, and the clay content is generally low. The above characteristics show that this area obviously has the characteristics of near provenance and rapid deposition.

\section{Diagenesis}

\subsection{Compaction}

Compaction includes early mechanical compaction and late pressure solution. It is dominated by early mechanical compaction, and late pressure solution is not obvious. Under the polarized light microscope, mechanical compaction is mainly manifested in point and line contact between rigid particles, and deformation between plastic particles due to suture contact, which is mainly manifested as the flake mineral biotite being squeezed and crushed. During the diagenesis process, cementation hinders the further compaction of the particles. Tuff cuttings are mainly crystal cuttings tuff and vitreous cuttings tuff. The internal mechanical compaction of the cuttings is strong, which is manifested by the brittle fracture of the crystal cuttings and the bump contact and suture contact between the plastic cuttings. The mechanical compaction of sintered tuff is manifested in the phenomenon of pseudorhyolite structure and fusion welding of cemented lava.

\subsection{Silica cementation}

The silica gel complex can be expressed in two forms: crystalline and amorphous. The amorphous-us is opal and the crystalline is quartz. The opal cements in the pyroclastic sandstone are widely distributed, apparently from the alteration of volcanic glass. Because the precipitation of opal is relatively fast, it is difficult for the opal supersaturated water to stay in a stable state for a long time or to migrate over long distances. The source of its silica may be nearby volcano Clastic.

\subsection{Carbonate cementation}

Carbonate cementation is due to the tectonic origin of the late diagenesis carbonate solution filling the intergranular fractures between gravel particles.

\subsection{Recrystallization}

The polymorphic transformation of minerals is more complex and broader recrystallization. Under normal circumstances, when one mineral transforms into another more stable mineral phase, only changes in crystal lattice, shape and size occur. During the burial and diagenesis of pyroclastic sedimentary rocks in Udert area, the most common is the transformation of opal from amorphous silica to chalcedony and quartz. Among them, the opal of amorphous silica comes from the devitrification process of volcanic glass.

\subsection{Accountability}

Metasomatism refers to the replacement of minerals by new minerals at the same time as the minerals are dissolved (mineral replacement). The newly formed minerals do not have the same chemical composition as the dissolved minerals, but often have the morphological characteristics of the original minerals, such as calcite metasomatic feldspar. The formation of authigenic minerals related to cementation and metasomatism include alteration and recrystallization. The effect of thin slices in this area is not obvious under the microscope.

\section{Pore type}

Affected by different diagenesis, pore types are diverse ${ }^{[8]}$. Pores can be divided into primary pores, secondary pores, and microcracks according to their genesis. Primary pores mainly refer to the pores between clastic particles formed by sedimentation, and secondary pores refer to the pores and fracture caves formed in the rock due to diagenesis such as dissolution and metasomatism after the formation of sedimentary rocks. The pores under the thin-slice lens in this area are mostly intergranular pores, that is, the volcanic clastic rock supported by clastic particles, the original pores left by the clastic particles are not filled by miscellaneous bases, and the content of cement is small.

\subsection{Primary pores}

It mainly includes normal intergranular pores and residual intergranular pores. Residual intergranular pores refer to intergranular pores that are not completely cemented. The primary intragranular pores are mainly the pores in the cuttings or the pores in the extruded rock cuttings, which are derived from the parent rock. Mineral cleavage joints mainly refer to flake or wedge-shaped cleavage joints common in minerals such as feldspar and mica, and their width is less than $0.1 \mu \mathrm{m}$. The surface of quartz crystal chips will also leave blast scar pores due to volcanic eruption. Although such pores can be filled by artificial castings, they are generally ineffective pores that do not contain hydrocarbons and contribute little to permeability. But it can dissolve along the cleavage seam and surface.

\subsection{Secondary pores}

The dissolution in this area is weak, manifested by the metasomatism of calcite crystals and dolomite crystals and dawsonite crystals. The metasomatic distribution of calcite crystals is wide, dolomite crystals are mostly developed in local areas, and dawsonite metasomatizes matrix and detrital particles at the same time. Due to the migration of soluble substances, the pores are mostly intergranular dissolved pores. 


\subsection{Micro cracks}

The microcracks are mostly filled with siliceous or partly filled with siliceous. When silicon fills the cracks completely, the cracks become invalid. When there is no silicon filling or partial filling, it is an effective crack. There are few effective cracks seen in the thin slices in this area, and the cracks are generally dominated by a main crack, accompanied by small sub-parallel cracks. Its contribution to permeability is relatively large, mainly due to structural causes.

\section{Conclusion}

(1) The main types of diagenesis are compaction, siliceous cementation and recrystallization, of which siliceous cementation and recrystallization play the main role, and compaction only works in the early stage.

(2) Pore types include normal intergranular pores and residual intergranular pores of primary pores, intern granular pores of secondary pores, and microcracks.

\section{References}

1. Cui Xin, Li Jianghai, Jiang Hong Fu, Wang Yun Zeng, Qi Linhai, Yang Shaoying."Superimposed" depositional style of fan delta in fault basin and its oil and gas geological significance-Xing'anling Formation in Sudeerte structural belt in Hailar Basin Examples of I and II oil groups[J]. Natural Gas Geoscience,2018,29(05):682-695.

2. Zhang Peng. Research on fine seismic interpretation and reservoir prediction of Xing'anling oil layer in Sudeerte area[D]. Northeast Petroleum University, 2017.

3. Su Xiangguang, Sun Yiling. Analysis of the control effect of fractures on the distribution of oil and water in buried hill reservoirs: A case study of Budate Group buried hill reservoirs in Sudeerte Oilfield, Hailar Basin[J]. Petroleum Geology and Engineering,2019, 33(05): 11-14+20.

4. Cui Xin, Li Jianghai, Wang Yunzeng, Liu Zhonglan, Zhang Hongwei, Qi Linhai.Characteristics and controlling factors of basement fractures in Sudeerte structural belt in Hailar Basin[J]. Geological Review,2016, 62(05): 1257-1270.

5. Wang Zhiqiang, Liu Bo, Hu Zhongyi, Wang $\mathrm{Xu}, \mathrm{Li}$ Junhui.High-resolution sequence stratigraphic framework and sedimentary characteristics of Nantun Formation in Sudeerte area, Hailar Basin[J]. Daqing Petroleum Geology and Development, 2019, 38(04):7-14.

6. Ma Yao, Li Wenhou, Wang Ruogu, Zhang Huiruo. The diagenesis and quantitative analysis of diagenetic facies of reservoirs in the 2 3 member of Shan_2 3 in the northern Zizhou gas field of Ordos Basin[J]. Natural Gas Geoscience, 2015 ,26(11): 2039-2052.

7. Tian Yanhong, Liu Shugen,Zhao Yihua, Song Jinmin, Song Linke, Sun Wei, Liang Feng, Zhang Changjun,
Li Junliang, Yin Kewei, Wang Chenxia, Wu Juan, Lin Tong,Bai Zhiqiang, Peng Hanlin,Chen Huizhi.Diagenesis of the Longwangmiao Formation in the Central Sichuan Basin[J]. Journal of Chengdu University of Technology (Natural Science Edition), 2014,41(06): 671-683.

8. Zhao Difei, Guo Yinghai, Yang Yujuan, Wang Shouyu, Mao Xiaoxiao, Li Mi. Diagenesis of Lower Silurian Longmaxi Formation Shale Reservoir in Southeastern Chongqing and Its Impact on Pore Development[J]. Journal of Palaeogeography, 2016 ,18(05): 843-856. 